\title{
Contributions to the knowledge autumnal lepidoptera species of Dağlıca (Hakkari, Southeastern Turkey) with two poorly known noctuid moths
}

\author{
Erdem Seven \\ Department of Gastronomy and Culinary Arts, School of Tourism and Hotel Management, Batman University, \\ Batman, 72060, Turkey \\ erdem_seven@hotmail.com
}

\begin{abstract}
This paper evaluates a collection of autumnal Lepidoptera species from Dağlica vicinity of Hakkari Province. A total of 58 species are examined belonging to 9 families. Off all, 33 species in Dağlıca vicinity and 21 species in Hakkari Province are determined for the first time. All the species are listed alphabetically within the families, together with synonyms and studied materials. Furthermore, distribution of endemic and poorly known two noctuid species, Ostheldera arne L. Ronkay \& Varga, 1994 and Hakkaria varga (Hacker, [1987]) are discussed and, their adults and genital pictures are illustrated.

Key words: Fauna, Lepidoptera, Dağlıca, Hakkari, Turkey.

\section{Özet}

$\mathrm{Bu}$ makale, Hakkari İlinin Dağlıca bölgesinin sonbahar Lepidoptera faunasına ait bir koleksiyonunu değerlendirmektedir. Toplamda, 9 familya içerisindeki 58 tür incelenmiştir. Bunlardan 33 tür Dağlıca'da, 21 tür Hakkari'de ilk kez tespit edilmiştir. Tüm türler familyaları içerisinde alfabetik olarak, sinonimleri ve incelenen örnekleri ile birlikte listelenmiştir. Bununla birlikte, endemik ve az bilinen iki noctuid türü, Ostheldera arne L. Ronkay \& Varga, 1994 ve Hakkaria varga (Hacker, [1987])'nın dağılışları tartışılmış, ergin ve genital resimleri eklenmiştir.
\end{abstract}

Anahtar kelimeler: Fauna, Lepidoptera, Dağlıca, Hakkari, Türkiye.

\section{Introduction}

Dağlica vicinity is located in Hakkari Province within the south-east of Turkey. It is very near to the northern border of Iraq and surrounded by steep mountains. Hakkari is an extremely mountainous region, and is one of the most challenging areas in Turkey regarding the studies on nocturnal Lepidoptera species due to security and geographical conditions.

Autumn species of Dağlica is sufficiently not known. However, some recent studies have attempted to useful in this respect. Kemal et al. $(2017,2018)$ are listed 189 spring species of 25 families in 2017 and subsequently, 390 species of 35 families from Dağlica vicinity in 2018. Furthermore, according to studies published at irregular intervals, totally 1012 Lepidoptera species were known in Hakkari Province (Koçak and Kemal 2015, 2018; Kemal and Koçak 2018). The results in this paper concluded that 21 species are new to fauna of Hakkari Province and 33 species are new to fauna of Dağlica vicinity. Moreover, endemic and poorly known two noctuid species, Hakkaria varga (Hacker, [1987]) (fig. 1) 
and Ostheldera arne L. Ronkay \& Varga, 1994 (fig. 2), which are active in autumn, have also been determined in the research region.

The genus Ostheldera Nye, 1975 is the sister group of Asteroscopus Boisduval, 1828 and comprises 6 species: O. gracilis (Osthelder, 1933); O. persa Ronkay \& Varga, 1994; O. arne Ronkay \& Varga, 1994; O. minna Ronkay \& Varga, 1994; O. kondara Varga \& Ronkay, 1991 and O. pakistana Ronkay, Ronkay \& Gyulai, 2011 (Ronkay et al. 2011). In Turkey, two of them, O. arne L. Ronkay \& Varga, 1994 and O. gracilis (Osthelder, 1933) are known (Koçak and Kemal 2018).

Ostheldera arne L. Ronkay \& Varga, 1994 is known from a relatively small area extending from southeastern Turkey to north-west Iran. Type locality of the species is $5 \mathrm{~km}$ south of Hakkari, $1800 \mathrm{~m}$, holotype of male in coll. M. Fibiger (Ronkay et al. 2011). Hakkaria varga (Hacker, [1987]) was firstly described in Polymixis genus by Hacker in 1987. After that Ronkay and Varga (1990) transferred this species in a newly created genus "Hakkaria". The genus is represented by a single species which belongs to another phyletic line, being transitional between the generic groups Polymixis and Dasypolia (Ronkay and Varga 1990). It represents phyletic line of unique combination of features. Until now, the species is only known in its type locality that is Şırnak Province, Tanin Mountain, Elkek Passage, $7 \mathrm{~km}$ from Uludere, $2200 \mathrm{~m}, 15.09 .1985$ (leg. and coll. H. Hacker). After long years, the second locality of these rare species have been found in Dağlıca vicinity, Hakkari Province, south-easternmost of Turkey with this study.

\section{Material and Methods}

Samples were collected from Dağlıca vicinity of Hakkari Province on 19 September 2016, $1280 \mathrm{~m}$,

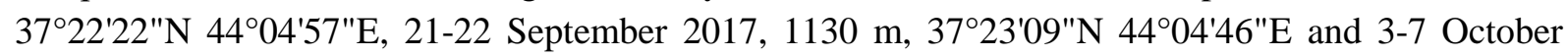
$2017,1350 \mathrm{~m}, 37^{\circ} 22^{\prime} 41^{\prime \prime} \mathrm{N} 44^{\circ} 05^{\prime} 05^{\prime \prime} \mathrm{E}$, in step areas containing sparse oaks, with a simple UV light trap system (UV strip led, 12V 7Ah battery and a transparent box). After field studies, pinned specimens were stretched and diagnosed according to these sources: De Freina and Witt (1987), Fibiger (1990, 1993, 1997), Ronkay and Varga (1990, 1994), Hesselbarth et al. (1995), Ronkay et al. (2001, 2011), Hausmann (2001, 2004), Hacker et al. (2002), Goater et al. (2003), Mironov (2003), Zilli et al. (2005), Fibiger and Hacker (2007), Leraut (2006, 2009), Fibiger et al. (2009, 2010), Hausmann and Viidalepp (2012). Identified materials are preserved in the Biology Laboratory of Batman University and special collection of the author in Batman Province.

In the results part, species are presented in alphabetical order within the families. New records in Dağlica vicinity are marked with an asterisk (*) symbol and likewise new records in Hakkari Province are marked with a plus $\left(^{+}\right)$symbol after the name of the species.

\section{Results \\ Butterflies:}

\section{Argynnidae}

Argynnis pandora ([Denis \& Schiffermüller], 1775)

Synonym(s): pandora [Denis \& Schiffermüller], 1775; maja Cramer, 1775 nec Fabricius, 1775; cynara Fabricius, 1777; cyrnea Schwerda, 1926; transcaucasica Moucha, 1967; deserticola Gross \& Ebert, 1975. Material studied: $3 \hat{\partial} \widehat{\partial}$, 19.IX.2016; $1 \hat{\partial}, 03 . X .2017$.

\section{Satyridae}

Chazara briseis (Linnaeus, 1764) 
Synonym(s): briseis Linnaeus, 1764; daedale Bergsträßer, 1780; bataia Fruhstorfer, 1909; interjecta Verity, 1916; variabilis Varin, 1958; pictonica Varin, 1958. Material studied: 10, 22.IX.2017.

\section{Moths:}

\section{Arctiidae}

Axiopoena karelini Ménétriés, 1863*

Synonym(s): karelini Ménétriés, 1863; transcaucasia Sheljuzhko, 1926; manissadjiani O.Bang-Haas, 1927. Material studied: 1 , , 22.IX.2017.

\section{Ctenuchidae}

Dysauxes famula (Freyer, 1836)*

Synonym(s): famula Freyer, 1836; hyalina Freyer, 1845; ragusaria Zickert, 1904; herthina Stauder, 1921; burgeffi Draudt, 1931; taurica Draudt, 1931; pseudoservula Naufock, 1933; pontica Friese, 1959;

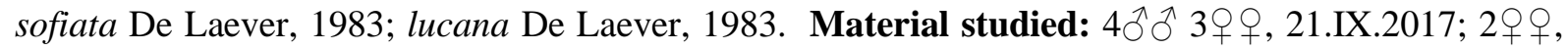
04-07.X.2017.

\section{Geometridae}

Aplocera plagiata (Linnaeus, 1758)

Synonym(s): plagiata Linnaeus, 1758; pallidata Staudinger, 1879. Material studied: $3 \hat{\jmath}$, 19.IX.2016; $1 \overbrace{}^{\Uparrow}$ 06.X.2017.

Apochima diaphanaria (Püngeler, 1904)

Synonym(s): diaphanaria Püngeler, 1904. Material studied: $5 \hat{\jmath} 3 \hat{\beta}++$, 22.09.217; $3 \hat{\jmath} 05-$ 07.X.2017.

Charissa dubitaria (Staudinger, 1892)*

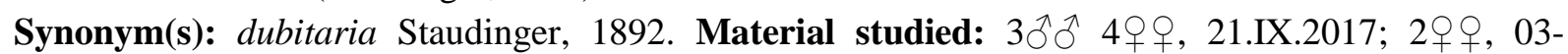
05.X.2017.

Charissa onustaria (Herrich-Schäffer, [1852])*+

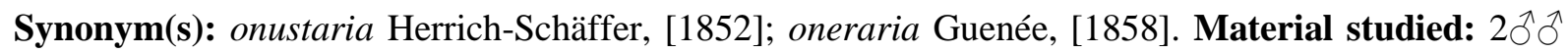
2우, 21.IX.2017.

Chesistege korbi (Bohatsch, 1910)

Synonym(s): korbi Bohatsch, 1910. Material studied: $3 \hat{\jmath} \widehat{\jmath}, 04 . X .2017$.

Coenotephria schneideraria (Lederer, 1855)*+

Synonym(s): schneideraria Lederer, 1855; taurica Staudinger, 1901; eteocretica Rebel, 1906. Material studied: $2 \widehat{\jmath} 4 q$ \% , 21.IX.2017; 5우, 06.X.2017.

Crocallis elinguaria (Linnaeus, 1758)*+

Synonym(s): elinguaria Linnaeus, 1758; trapezaria Boisduval, 1840. Material studied: $3 \hat{\jmath} \widehat{\delta} 5$ 우, 22.IX.2017; 2 우, 04-06-07.X.2017.

Ennomos quercarius (Hübner, [1813])*+

Synonym(s): quercaria Hübner, [1813]. Material studied: $2 \AA \widehat{\jmath} 1 q$, 04.X.2017. 
Eumera hoeferi Wehrli, 1934* +

Synonym(s): \#höferi Wehrli, 1934; hoeferi Wehrli, 1934; \#hoferi Wiltshire, 1937. Material studied: 2우, 21.IX.2017.

Glossotrophia sacraria (A.Bang-Haas, 1910)

Synonym(s): sacraria A.Bang-Haas, 1910. Material studied: $3 \widehat{\jmath} 5 q \propto+, 22 . I X .2017 ; 3 \widehat{\partial}$, 0506.X.2017.

Gnophos pseudosnelleni Rjabov, 1964*

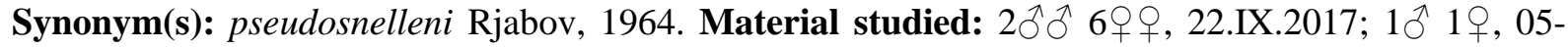
07.X.2017.

Idaea degeneraria (Hübner, [1799])

Synonym(s): degeneraria Hübner, [1799]; degenerata Treitschke, 1828; floridaria Turati, 1913. Material studied: $3 \widehat{\partial} 2$ 우 9 , 03-04.X.2017.

Odontognophos zacharius (Staudinger, 1879)*+

Synonym(s): zacharia Staudinger, 1879. Material studied: 1ठ઼, 03.X.2017.

Scopula immistaria (Herrich-Schäffer, [1852])

Synonym(s): \#elisabetharia Heydenreich, 1851; immistaria Herrich-Schäffer, [1852]. Material

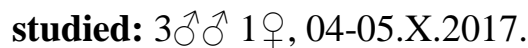

Scopula marginepunctata (Goeze, 1781)

Synonym(s): marginepunctata Goeze, 1781; conjugata Borkhausen, 1794; aniculosata Rambur, 1829; puellaria Boisduval, 1840; mutaria Heydenreich, 1851; promutata Guenée, [1858]; promutaria Morris, 1861; apertaria Walker, [1863]; madoniata Fuchs, 1901; pastoraria De Joannis, 1891. Material

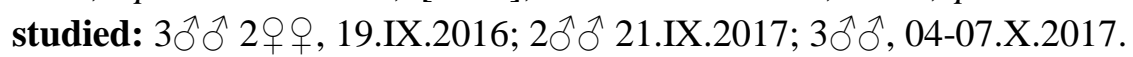

Lasiocampidae

Lasiocampa eversmanni (Kindermann, 1843)

Synonym(s): eversmanni Kindermann, 1843; eversmanni Freyer, 1844; attrita Stshetkin, 1960. Material studied: $3 \widehat{\partial} 2$ 2ㅇ, 21.IX.2017; 3ठึ, 03-04.X.2017.

Lasiocampa grandis (Rogenhofer, 1891)

Synonym(s): grandis Rogenhofer, 1891. Material studied: $2 \hat{\jmath} \widehat{\partial} 1$ ㅇ, 22.IX.2017; $3 \hat{\partial} \widehat{\partial}, 04 . X .2017$.

Trichiura stroehlei Zolotuhin, 2007*

Synonym(s): stroehlei Zolotuhin, 2007. Material studied: $2 \widehat{\partial}$, 04.X.2017.

\section{Lemoniidae}

Lemonia pia Püngeler, 1902

Synonym(s): pia Püngeler, 1902. Material studied: $3 \hat{\jmath} 1$ 1 + , 05.X.2017.

\section{Noctuidae}

Agrochola lactiflora (Draudt, 1934)*+

Synonym(s): lactiflora Draudt, 1934; fibigeri Hacker \& Moberg, 1989. Material studied: $3 \lesssim \widehat{\jmath} 1$, 04-05.X.2017. 
Agrochola humilis (Fabricius, 1787)*

Synonym(s): \#humilis [Denis \& Schiffermüller], 1775; humilis Fabricius, 1787. Material studied: 2웅, 03.X.2017.

Allophyes renalis (Wiltshire, 1941)*

Synonym(s): renalis Wiltshire, 1941; crassicornis Brandt, 1941. Material studied: $2 \precsim ð$, 05.X.2017.

Ammoconia caecimacula (Fabricius, 1787)*+

Synonym(s): caecimacula Fabricius, 1787; millegrana Esper, [1790]; respersa Brahm, 1791;

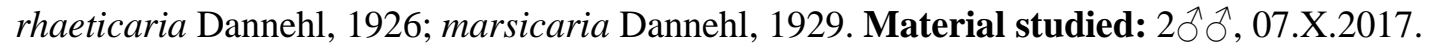

Autophila ligaminosa (Eversmann, 1851)*+

Synonym(s): ligaminosa Eversmann, 1851; caucasica Herz, 1904. Material studied: $3 \hat{\delta} \widehat{\partial} 2$ 우웅,

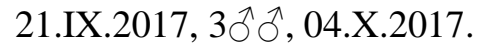

Catocala elocata (Esper, [1787])

Synonym(s): elocata Esper, [1787]; marita Hübner, [1813]; nurus Hübner, [1822]. Material studied: 1ठิ, 22.IX.2017.

Chersotis margaritacea (Villers, 1789)*+

Synonym(s): margaritacea Villers, 1789; i-intactum Hübner, [1803]; \#abruzzensis Corti \& Draudt, 1933; espunensis Calle, 1982. Material studied: $2 \widehat{\jmath} \sigma^{\Uparrow} 1$ ㅇ, 03-05.X.2017.

Chersotis obnubila (Corti, 1926)*

Synonym(s): obnubila Corti, 1926; maraschi Corti \& Draudt, 1933 (p.61) nec Corti \& Draudt, 1933

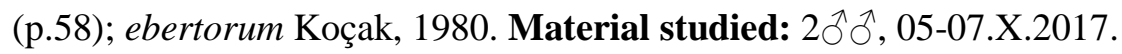

Dichagyris amoena (Staudinger, 1892)

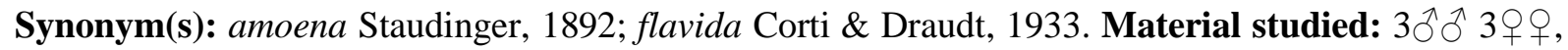
03-04.X.2017.

Dichagyris fredi (Brandt, 1938)

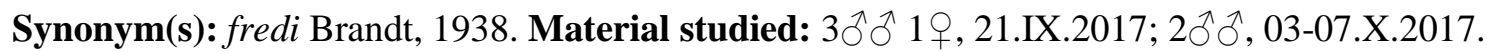

Dichagyris singularis (Staudinger, 1877)

Synonym(s): singularis Staudinger, 1877. Material studied: $11 \hat{\jmath} \widehat{\delta}$ 5q⿱中, 19.IX.2016, $3 \hat{\jmath} \widehat{\partial}, 21-$

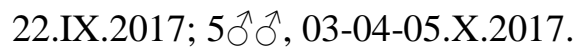

Dichagyris nachadira (Brandt, 1941)*+

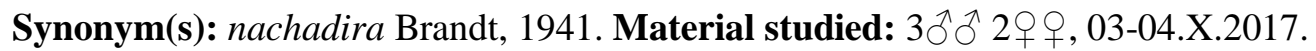

Dichonia aeruginea (Hübner, [1803])* +

Synonym(s): aeruginea Hübner, [1803]; mioleuca Geyer, [1828]; chioleuca Treitschke, 1835; mesembrina Schawerda, 1913. Material studied: $3 \widehat{\jmath} \widehat{\partial}, 04-05 . X .2017$. 
Dryobotodes carbonis (F.Wagner, 1931)*+

Synonym(s): carbonis F. Wagner, 1931; taurica Osthelder, 1933; europaea Pinker, 1976. Material studied: $2 \hat{\jmath} \widehat{\partial} 1$ ㅇ, 05-06.X.2017.

Episema korsakovi (Christoph, 1885)

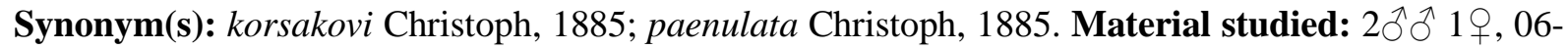
07.X.2017.

Eugnorisma enargiaris (Draudt, 1936)

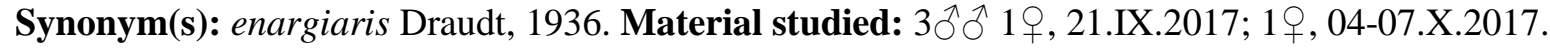

Gortyna flavago ([Denis \& Schiffermüller], 1775)*+

Synonym(s): flavago [Denis \& Schiffermüller], 1775; ochracea Hübner, 1786; lappae Donovan, 1801; ochraceago Howarth, 1809; cinerea Goossens, 1880. Material studied: $2 \hat{\jmath} \widehat{\partial}, 04 . X .2017$.

Hakkaria varga (Hacker, [1987])*+(fig. 1)

Synonym(s): varga Hacker, [1987]. Material studied: $1 \hat{\jmath}$, 03.X.2017, slide no. 576ðे.

Helicoverpa armigera (Hübner, [1808])

Synonym(s): obsoleta auct. nec Fabr., 1775; barbara Fabricius, 1794 [rejected]; armigera Hübner, [1808]; pulverosa Walker, [1857]; uniformis Wallengren, 1860; rama Bhattacherjee \& Gupta, 1972. Material studied: $2 \hat{\jmath} \partial^{\lambda}, 06-07 . X .2017$.

Hoplodrina ambigua ([Denis \& Schiffermüller], 1775)

Synonym(s): ambigua [Denis \& Schiffermüller], 1775; plantaginis Hübner, [1813]; uniformis Swinhoe, 1885. Material studied: $7 \lesssim \widehat{\jmath} 3$ 우 0 , 22.IX.2017; 2우, 03-07.X.2017.

Lacanobia thalassina (Hufnagel, 1766)*+

Synonym(s): thalassina Hufnagel, 1766; humeralis Haworth, 1809; achates Hübner, [1813]. Material studied: $2 \widehat{\jmath}, 06-07 . X .2017$.

Luperina rjabovi (Kljutschko, 1967)* +

Synonym(s): rjabovi Kljutschko, 1967. Material studied: 4 ô $\widehat{\jmath} 1$ 1 + , 03-07.X.2017.

Melanchra persicariae (Linnaeus, 1761)*

Synonym(s): persicariae Linnaeus, 1761; sambuci Hufnagel, 1766; graphica Geoffroy, 1785; accipitrina Esper, [1788]. Material studied: $2 \widehat{\partial}, 03 . X .2017$.

Metopodicha ernesti Draudt, 1936*

Synonym(s): ernesti Draudt, 1936; achaemenica Wiltshire, 1941. Material studied: $3 \hat{\jmath} \widehat{\delta} 1$ q 22.IX.2017; 1 , , 03.X.2017.

Ostheldera arne L. Ronkay \& Varga, 1994* (fig. 2)

Synonym(s): arne L. Ronkay \& Varga, 1994. Material studied: $1 \overbrace{}^{\Uparrow} 1$, 04.X.2017; slide no. 575 581 . 
Oxytripia stephania Sutton, [1964]

Synonym(s): stephania Sutton, [1964]; danilevskyi Miljanovsky, 1973. Material studied: $2 \widehat{\partial}$, 04.X.2017.

Polymixis rufocincta (Geyer, 1828)*+

Synonym(s): rufocincta Geyer, [1828]; farinosa Freyer, 1848; mucida Guenée, 1852. Material studied: $3 \hat{\jmath} \widehat{\partial}, 21 . I X .2017 ; 1$,, $06 . X .2017$.

Polymixis manisadjiani (Staudinger, 1881)

Synonym(s): manisadjiani Staudinger, 1881. Material studied: $4 \widehat{\jmath}$, 19.06.2016; 1ภ, 22.IX.2017.

Polymixis rosinae (Bohatsch, 1910)*+

Synonym(s): rosinae Bohatsch, 1910; paradisiaca Boursin, 1944; gilva Sukhareva, 1976; paravarga L.Ronkay, 1990; himalaya Hacker \& Weigert, 1990. Material studied: 1ठ 2우, 04.X.2017.

Pseudenargia regina (Staudinger, 1892)*+

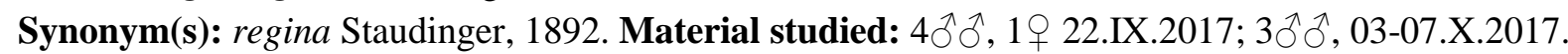

Scotochrosta pulla ([Denis \& Schiffermüller], 1775)

Synonym(s): \#pulla [Denis \& Schiffermüller], 1775. Material studied: $2 \hat{\jmath}, 06 . X .2017$.

Xanthia cypreago (Hampson, 1906)* +

Synonym(s): cypreago Hampson, 1906. Material studied: $3 \hat{\jmath} \partial^{\hat{\alpha}}$, 05-07.X.2017.

Zethes brandti Janzon, 1977

Synonym(s): nemea Brandt, 1938 (p.p.); nemea sensu Koçak, 1977; brandti Janzon, 1977. Material studied: $1 \hat{\jmath}, 06 . X .2017$.

\section{Saturniidae}

Saturnia caecigena Kupido, 1825

Synonym(s): caecigena Kupido, 1825; unicolor Schultz, 1910; wiskotti Niepelt, 1914; derosata

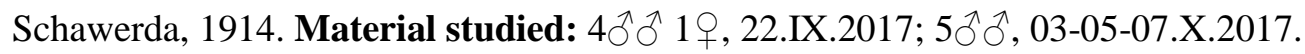

Saturnia naessigi (De Freina, 1992)*

Synonym(s): naessigi De Freina, 1992. Material studied: 5ðð 2q $q$, 05-06-07.X.2017.

\section{Discussion and Conclusion}

This study contributes to Lepidoptera species of Dağlıca vicinity (Hakkari), which is one of the hardest region in Turkey because of geographical and security conditions. And, the research will also contribute to the determining of Lepidoptera fauna of Turkey.

In this paper, autumnal Lepidoptera species of Dağlıca vicinity are examined faunistically. Totally, 58 species are evaluated belonging to Argynnidae (1), Satyridae (1) and, Arctiidae (1), Ctenuchidae (1), Geometridae (15), Lasiocampidae (3), Lemoniidae (1), Noctuidae (33), Saturniidae (2) families. Among them, 33 species are determined as new to the fauna of Dağlica vicinity and 21 species are detected as new to the fauna of Hakkari Province. A large part of the collected species are composed of Noctuidae and Geometridae families. Thus, the number of Lepidoptera species reached 1033 in Hakkari Province. 
Moreover, distributionally endemic and poorly known Hakkaria varga (Hacker, [1987]) (fig. 1) and Ostheldera arne L. Ronkay \& Varga, 1994 (fig. 2) species are assessed. O. arne was known a small area from south-eastern Turkey (Hakkari) to north-west Iran (Miyane, Azerbaijan Prov.) (Ronkay et al. 2011). H. varga was known only type locality, Şırnak Province (Turkey) so far (Hacker 1987). The second locality of these rare species have been discovered in Dağlıca vicinity, near to the type localities of the species. Both species are distributed in a very limited area and their populations are very lowdensity. By this way, these new data also contribute to the spreading area of the species.
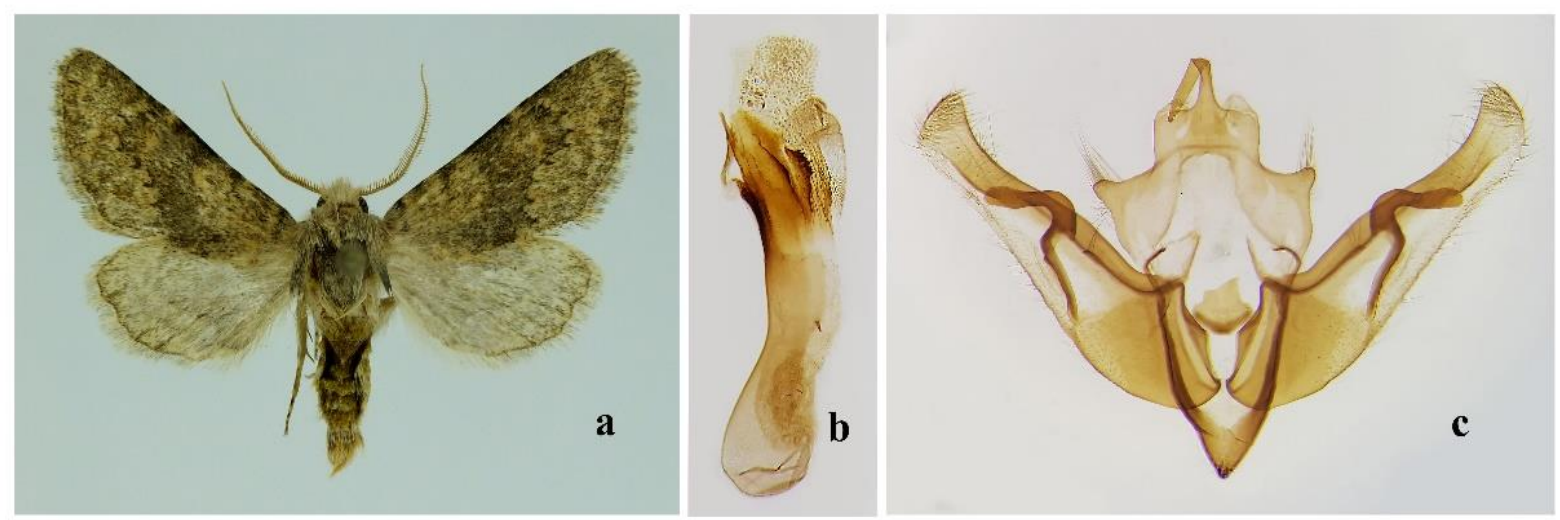

Figure 1. Hakkaria varga, a. adult male, b-c. male genitalia (slide no. 576§ึ), b. aedeagus, c. genital capsule.

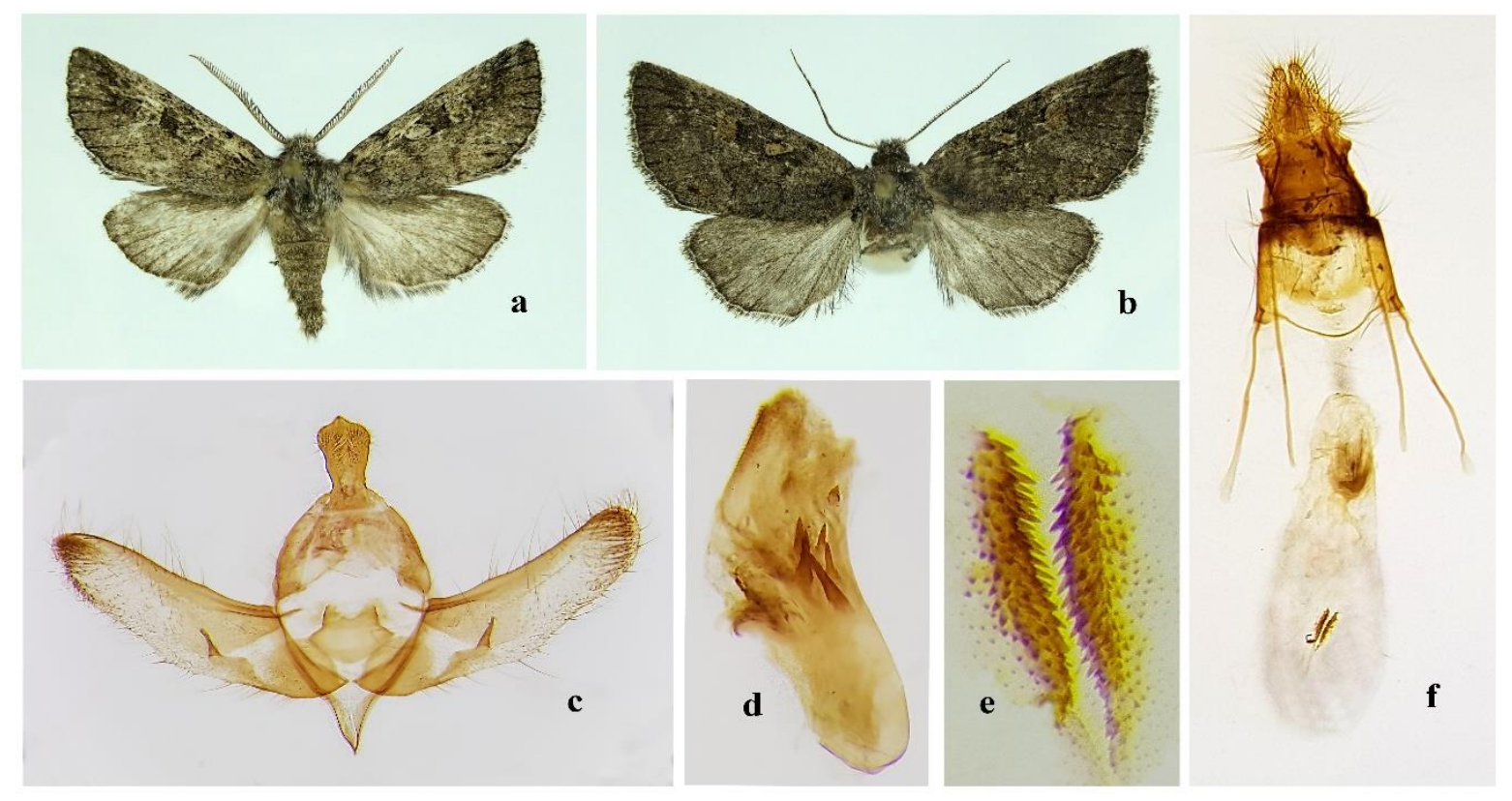

Figure 2. Ostheldera arne, a. adult male, b. adult female, c-d. male genitalia (slide no $575 \AA$ ), c. genital capsule, d. aedeagus, e. signum, f. female genitalia (slide no. 581 \%).

\section{Acknowledgments}

The author would like to thank Doğan YOLBAŞ for help in collecting of the samples.

\section{References}

De Freina, J., Witt, T. (1987). Die Bombyces und Sphinges der Westpalaerktis. Band 1: Nolidae, Arctiidae, Syntomidae, Dilobidae, Lymantriidae, Notodontidae, Thaumetopoeidae, Thyretidae, Axiidae, Drepanidae, 
Thyatiridae, Bombycidae, Brahmaeidae, Endriomidae, Lasiocampidae, Lemoniidae, Saturniidae, Sphingidae. 47 Farbtafeln, 330 Karten; München, pp, 708.

Fibiger, M. (1990). Noctuidae Europaeae. Vol. 1, Noctuinae I, Entomological Press, Soro, Denmark, pp, 208.

Fibiger, M. (1993). Noctuidae Europaeae. Vol. 2, Noctuinae II, Entomological Press, Soro, Denmark, pp, 230.

Fibiger, M. (1997). Noctuidae Europaeae. Vol. 3, Noctuinae. Entomological Press, Soro, Denmark, pp, 418.

Fibiger, M., Hacker, H. (2007). Noctuidae Europaeae. Vol. 9, Amphipyrinae, Condicinae, Eriopinae, Xyleninae. Entomological Press, Soro, Denmark, pp, 410.

Fibiger, M., Ronkay, L., Steiner, A., A, Zilli. (2009). Noctuidae Europaeae. Vol. 11, Pantheinae, Dilobinae, Acronictinae, Eustrotiinae, Nolinae, Bagisarinae, Acontiinae, Metoponiinae, Heliothinae and Bryophilinae. Entomological Press, pp, 504.

Fibiger, M., Ronkay, L., Yela, J.L., A, Zilli. (2010). Noctuidae Europaeae. Vol. 12, Rivulinae, Boletobiinae, Hypenodinae, Araeopteroninae, Eublemminae, Herminiinae, Hypeninae, Phytometrinae, Euteliinae and Micronoctuidae. Inluding Supplement to Volumes 1-11. Entomological Press, pp, 451.

Goater, B., Ronkay, L., Fibiger, M. (2003.) Noctuidae Europaeae. Vol. 10, Catocalinae, Plusiinae. Entomological Press, pp, 452.

Hacker, H. (1987). Fünfter beitrag zur systamatik Erfassung der Noctuidae der Türkei. Beschreibung neuen taxa und faunistisch bemerkenwerte funde aus den aufsammlungen von Wolf und Hacker aus dem Jahr 1985 sowie erganzun-gen zu früheren arbeiten (Lepidoptera). Atalanta, 17:27-83.

Hacker, H., Ronkay, L., Hreblay, M. (2002). Noctuidae Europaeae. Vol. 4, Hadeninae I. Entomological Press, pp, 419.

Hausmann, A. (2001). The Geometrid Moths of Europe Vol. 1, 8 Pls. Apollo Books, pp, 282.

Hausmann, A. (2004). The Geometrid Moths of Europe Vol. 2 Sterrhinae. Apollo Books, pp, 600.

Hausmann, A., J, Viidalepp. (2012). The Geometrid Moths of Europe. Volume 3., Apollo Books, Stenstrup, pp, 743.

Hesselbarth, G., Oorschot H.V., Wagener, S. (1995). Die Tagfalter der Türkei unter Berücksichtigung der angrenzenden Länder. Selbstverlag S.Wagener, Bocholt. Bde. 1 \& 2: 1354 S. Bd.3: pp, 847.

Kemal, M., Koçak, A.Ö. (2018). First faunistic results of the Lepidoptera of Zap Valley in Hakkari Province (SE Turkey). Priamus $16(4): 119-199$.

Kemal, M., Koçak, A.Ö., Uçak, H. (2017). On a collection of Lepidoptera from Dağlıca (South-East Turkey, Hakkâri Province). Cesa News 139:1-24.

Kemal, M., Koçak, A.Ö., Uçak, H. (2018). List of the Lepidoptera species of Dağlıca (Hakkari Province, SE Turkey). Cesa News 159:1-21.

Koçak, A.Ö., Kemal, M. (2015). Annotated List of the Lepidoptera of Hakkâri (SE Turkey). Cesa News 116:1146.

Koçak, A.Ö. Kemal M (2018). A synonymous and distributional list of the species of the Lepidoptera of Turkey.'” 
Cent. ent. Stud., Memoirs 8:1-487.

Leraut, P. (2006). Moths of Europe, volume 1, Saturnids, Lasiocampids, Hawkmoths, Tiger Moths. N. A. P. Editions, pp, 387.

Leraut, P. (2009). Moths of Europe. Volume 2 Geometrid moths. N.A.P. editions, pp, 808.

Mironov, V. (2003) The Geometrid Moths of Europe Vol. 4, Larentiinae II. Apollo Books, pp, 462.

Ronkay, L., Varga, Z. (1990). Taxonomic and zoogeographical studies on the subfamiliy Cuculliinae (Lepidoptera, Noctuidae) II Esperiana 1:471-497.

Ronkay, G., Ronkay, L., Gyulai, P. (2011). Cucullinae II and Psaphidinae. A taxonomic atlas of the Eurasian and North African Noctuoidea. Vol. 5. Heterocera Press, Budapest, pp, 380.

Ronkay, L., Varga, Z. (1994). On the taxonomy of the genus Ostheldera Nye, 1975 (Lepidoptera, Noctuidae, Cuculliinae). Acta Zoologica Academiae Scientiarum Hungaricae 40 (2):157-170.

Ronkay, L., Yela, J.L., Hreblay, M. (2001). Noctuidae Europaeae. Vol. 5, Hadeninae II. Entomological Press, Soro, Denmark, pp, 452.

Zilli, A., Ronkay, L., Fibiger, M. (2005). Noctuidae Europaeae: Apameini. Vol. 8, Entomological Press, Soro, Denmark, pp, 323.

Submitted: 01.10.2018

Accepted: 26.12.2018 